\title{
Ocular changes resulting from therapy with amiodarone
}

\author{
D. V. INGRAM, N. S. V. JAGGARAO, AND D. A. CHAMBERLAIN \\ From the Royal Sussex County Hospital, Brighton, and Sussex Eye Hospital, Brighton
}

SUMmARY Amiodarone, a powerful antiarrhythmic agent recently made available in Britain, is known to cause corneal changes, but the clinical implications of this unwanted effect are still controversial. We have made serial observations on 105 patients treated with the drug for periods ranging from 3 months to over 7 years. Corneal abnormalities were detected by slit-lamp examination in 103 patients $(98 \%)$. These always progressed over several months but subsequently showed a stable pattern which changed only with alteration of dose. The abnormalities regressed and disappeared within 7 months in the 16 patients whose treatment was discontinued for reasons unconnected with ocular changes. No macular changes or permanent sequelae occurred. Ocular symptoms were unusual: 6 patients had reactions in the skin of the eyelids, and 6 others had minor symptoms related to the corneal changes. We do not believe that ophthalmological surveillance is mandatory in asymptomatic patients on long-term amiodarone therapy.

Amiodarone is a potent antiarrhythmic agent which has been used widely in Europe since 1967. ${ }^{1}$ It has only recently been licensed in Britain for limited indications. Though acute toxicity does not readily occur during oral administration, the drug does have some unwanted effects during long-term administration. Among these the tendency to cause corneal deposits has aroused most concern. Ocular change resulting from amiodarone was first noted by Joseph and Rousselie in $1967 .{ }^{2}$ Some authors believe that all patients on maintenance treatment will show changes in due course. ${ }^{3}$ The estimates, however, of the percentage of patients showing corneal deposits have varied widely. ${ }^{45}$ We now report the ophthalmic observations made during a systematic follow-up of 105 patients treated with amiodarone for periods ranging from 3 months to over 7 years.

\section{Patients and methods}

The patients were treated with oral amiodarone in one cardiac clinic, though in a few instances the drug therapy had been initiated elsewhere. Their ages when first examined ranged from 23 to 89 years (median 63 years).

Dosage of amiodarone depended upon clinical need. The usual regimen was $200 \mathrm{mg}$ t.d.s. for one week, $200 \mathrm{mg}$ b.d. for one week, and 200 to $300 \mathrm{mg}$

Correspondence to Mr D. V. Ingram, FRCS, 4 Tongdean Road, Hove, Sussex BN3 6QB. daily thereafter. The exceptions were 19 patients maintained on doses ranging from $200 \mathrm{mg} 2$ to 5 times weekly, and 15 patients maintained on 400 to $600 \mathrm{mg}$ daily.

Most patients were seen first within 3 months of starting treatment. We arranged follow-up examinations at intervals not greater than 6 months both for those who remained on treatment and for patients whose therapy had been discontinued. The median duration of treatment was 1 year 11 months (range 7 years 3 months to 3 months). Seventy-four patients were followed up for at least a year after starting the drug.

At each visit patients were asked to volunteer any complaints referrable to their eyes, but direct questions on specific symptoms were avoided. Visual acuity was assessed by means of a conventional Snellen's chart, slit-lamp biomicroscopy was performed, and routine fundal examination was carried out. When relevant, detailed tests were made of tear secretion by Schirmer's method and of intraocular pressure by Goldmann's applanation tonometry. Colour vision was assessed in 14 patients by the Ishihara isochromatic plates.

\section{Results}

The symptoms reported by patients are listed in Table 1. Only haloes and photophobia were thought to be due to drug-induced ocular changes. Lid irritation 
Table 1 Ocular symptoms in 105 patients treated with amiodarone. No patient complained of more than one symptom.

\begin{tabular}{ll}
\hline Symptom & No. of patients \\
\hline Lid irritation & 6 \\
Photophobia & 3 \\
Haloes & 2 \\
Blurring of vision & 1 \\
\hline
\end{tabular}

was more common, but was thought to depend upon changes induced in the skin of the lid rather than in the eye itself. It was associated with a gritty sensation in 2 of the 6 patients affected. No objective findings were present in the patient with blurring of vision, and we doubt if this was a true effect of amiodarone.

No decrease in visual acuity attributable to the drug was observed. Fig. 1 compares visual acuity for each patient at the initial and most recent examination. No drug effect was observed on funduscopic examination with the possible exception of a chorioretinal haemorrhage in one patient: this may have resulted from an abnormal bleeding tendency as a result of an amiodarone-warfarin interaction.

Slit-lamp examination showed corneal abnormalities in 103 of 105 patients $(98 \%)$. The earliest changes comprised faint grey granular lines radiating from a median point at the junction of the middle and lower thirds of the cornea (Fig. 2). These resemble Hudson-Stahli lines. As the changes developed, the number and density of the lines increased. The most advanced abnormality had numerous curved dense

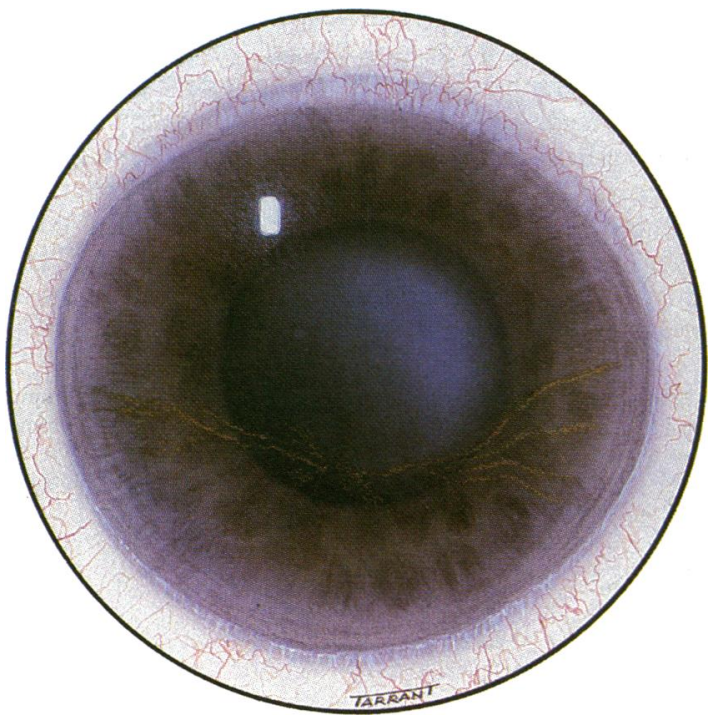

Fig. 2 Corneal abnormalities: earliest changes.

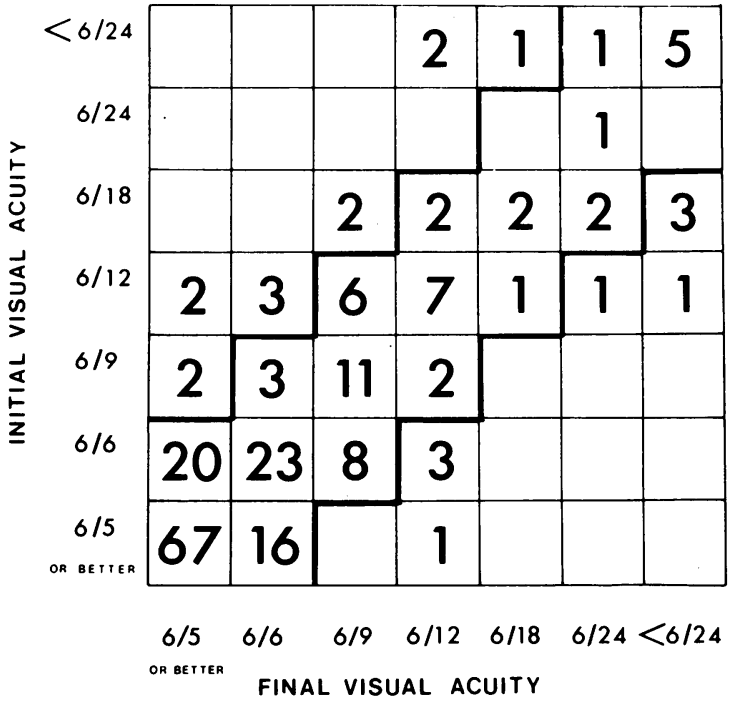

Fig. 1 Comparison of corrected visual acuity of 99 patients (198 eyes) at initial and final examinations while on treatment with amiodarone. All observations between heavy lines are considered to show no definite difference owing to the subjective nature of the Snellen test. The observations above the heavy lines show apparent improvement, and those below the heavy lines apparent deterioration. No systematic trend emerged.

lines forming whorled patterns, with uniform granular opacities involving the whole corneal epithelium (Fig. 3). Within a few months a stable pattern was established, which changed only with

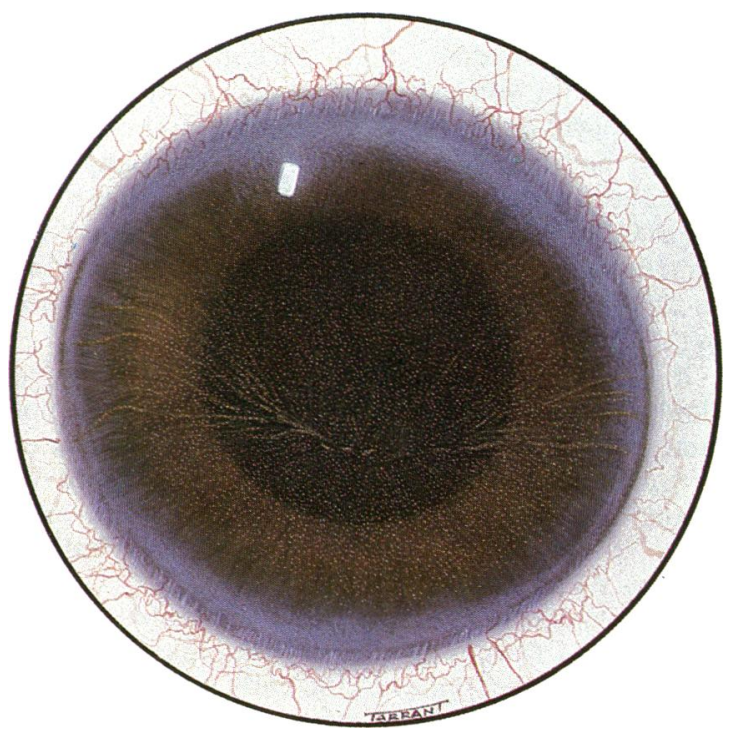

Fig. 3 Corneal abnormalities: advanced changes. 
alteration of dose. Deposits became denser if dosage was increased and regressed if dosage was reduced. The dose of amiodarone was reduced in 2 patients in whom these changes were especially marked.

Watillon et al. ${ }^{6}$ divided the changes into 3 stages according to their distribution, but the gradual progression renders precise classification difficult and of little practical value; grading was therefore not attempted in this series. The patterns were similar in the 2 eyes in the absence of other corneal pathology. Two patients with contact lenses developed characteristic abnormalities. Three patients had evidence of diminished tear secretion but no relationship to drug therapy was established. No rise in intraocular pressure was observed during treatment, and no changes were found in tests of colour vision.

The drug was withdrawn in 16 patients for reasons unconnected with the corneal deposits. The pattern always cleared over a period of 3 to 7 months, and no permanent corneal changes were observed which could have been attributable to the drug.

\section{Discussion}

At present amiodarone is generally available only for patients with disorders of heart rhythm associated with Wolff-Parkinson-White syndrome and for those with serious arrhythmias refractory to other conventional agents. Already, however, more than 5000 patients in Britain are on long-term oral therapy with amiodarone, and the number is likely to increase as the potency of its antiarrhythmic action becomes more widely appreciated. Unwanted effects are therefore a matter of wide concern. That the drug induces corneal changes is well known, but the cause remains speculative and-except possibly in France-little information is available on the longterm implications of the phenomenon. Following the initially unexpected problems with chloroquine ${ }^{7}$ and practolol, ${ }^{8}$ drugs with ocular side effects tend to be regarded with suspicion. Indeed constraints have sometimes been placed on the use of amiodarone because of the effects on the eye, but our experience suggests that this caution may be unwarranted.

In the present series corneal changes were found in almost every patient treated with amiodarone for more than 3 months. Only 2 patients in our series were without them. One of these is known to have blood levels of amiodarone within the therapeutic range. Biomicroscopy is therefore of only limited value as a test of noncompliance.

The changes are nearly always invisible to the naked eye and require slit-lamp for ready identification. The resemblance of the early appearances to Hudson-Stahli lines may cause confusion even to experienced ophthalmologists, but once recognised assessment requires only the briefest examination.

The corneal appearances are usually thought to result from deposits of the drug or a metabolite ${ }^{3}$ in the deeper layers of the epithelium. The explanation seems plausible because amiodarone contains iodine, which tends to be concentrated in the lacrimal glands and secreted in the tears. Other drugs such as chloroquine, ${ }^{7}$ hydroxychloroquine, ${ }^{9}$ mepacrine,${ }^{10}$ and phenothiazines, ${ }^{11}$ which are also deposited in or affect the cornea, produce similar whorled patterns; these may result from an effect at the limbus on the centripetally migrating epithelial cells. ${ }^{12}$ Electron microscopy of the corneal epithelium in amiodarone keratopathy has shown complex lipid deposits within lysosome-like intracytoplasmic inclusions. These may result from interactions of the drug or its metabolites with host material. ${ }^{13}$

The changes regress if amiodarone is discontinued. In our series clearing was always complete within 7 months. This is reassuring for those supervising treatment with amiodarone and for patients themselves. The lack of sequelae and the reversibility of the changes imply that routine ophthalmological supervision is unnecessary for patients who are free of ocular symptoms. The reduction in dosage on ophthalmological grounds in 2 patients of the present series was in retrospect probably unnecessary.

Only 12 of our 105 patients complained of symptoms which we judged to be related to drug therapy. The most common was lid irritation, and this was probably due to the photosensitivity of the skin ${ }^{14}$ rather than to any complications in the eye itself. Photophobia and halo formation might have been more common if larger doses of the drug had been used. These symptoms can be especially troublesome at night when patients look towards localised areas of relative brightness such as street lamps and headlights. Visual acuity, judged by the Snellen charts, seems not to be affected by the corneal changes; moreover there is no evidence of any effect on macular function. Though amiodarone does have other unwanted effects which may be important, ${ }^{15}$ the ocular changes are rarely troublesome enough to influence management.

We thank Mrs E. A. Quinn, research assistant, and Mrs M. Mepham, cardiac technician, for their assistance with the documentation and record keeping for this study.

We acknowledge financial assistance from Labaz to enable Figs. 2 and 3 to be reproduced in colour.

\section{References}

1 Vastesaeger M, Gillot P, Rasson G. Etude clinique d'une nouvelle médication anti-angoreuse. Acta Cardiol (Brux) 1967; 22: 483-500.

2 Joseph E, Rousselie F. Round table seminar on amiodarone, 19 October 1968. Paris. Quoted by François J. ${ }^{3}$ 
3 François J. Cornea verticillata. Doc Ophthalmol 1969; 27: 235-50.

4 Babel J, Stangos N. Lésions oculaires iatrogènes; l'action d'un nouveau médicament contre l'angor pectoris. Arch Ophtalmol (Paris) 1970; 30: 197-208.

5 Verin $\mathrm{Ph}$, Gendre $\mathrm{Ph}$, Barchewitz G, et al. Thesaurismose cornéenne par amiodarone. Arch Ophtalmol (Paris) 1971; 31: 581-96.

6 Watillon M, Lavergne G, Weekers J. Corneal deposits due to amiodarone. Bull Soc Belg Ophtalmol 1968; 150: 715-26.

7 Hobbs HE, Calnan CD. The ocular complications of chloroquine therapy. Lancet 1958; i: 1207-9.

8 Wright $P$. Untoward effects associated with practolol administration: oculo-muco-cutaneous syndrome. Br Med J 1975; i: 595-8.

9 Shearer RV, Dubois EL. Ocular changes induced by long-term hydroxychloroquine (Plaquenil) therapy. Am J Ophthalmol 1967; 64: 245-52.

10 Mann I. 'Blue Haloes' in Atebrin workers. Br J Ophthalmol 1947; 31: 40-6.

11 Mathalone MBR. Eye and skin changes in patients treated with chlorpromazine. Br J Ophthalmol 1967; 51: 86-93.

12 Bron AJ. Vortex patterns of the corneal epithelium. Trans Ophthalmol Soc UK 1973; 43: 455-72.

13 D'Amico DJ, Kenyon KR, Ruskin JN. Amiodarone keratopathy. Drug-induced lipid storage disease. Arch Ophthalmol 1981; 99: 257-61.

14 Rosenbaum MB, Chiale PA, Halpern MS, et al. Clinical efficacy of amiodarone as an anti-arrhythmic agent. Am J Cardiol 1976; 38: 934-44.

15 Marcus FI, Fontaine GH, Frank R, Grosgogeat Y. Clinical pharmacology and therapeutic actions of the anti-arrhythmic agent amiodarone. Am Heart J 1981; 101: 480-93. 